\title{
Revisão de pesquisas sobre criatividade e envelhecimento
}

\author{
Research review on creativity and aging
}

Revisión de la investigación sobre creatividad y envejecimiento

Tatiana de Cássia NAKANO

Docente permanente do Programa de Pós-Graduação Stricto Sensu em Psicologia, Pontifícia Universidade Católica de Campinas (Puc-Campinas) 13087-571 Campinas - SP, Brasil https://orcid.org/0000-0002-5720-8940

Janaina CHNAIDER

Doutoranda, Programa de Pós-Graduação Stricto Sensu em Psicologia, Pontifícia Universidade Católica de Campinas (Puc-Campinas) 13087-571 Campinas - SP, Brasil https://orcid.org/0000-0001-5567-6698 Isabel Cristina Camelo de Abreu Doutoranda, Programa de Pós-Graduação Stricto Sensu em Psicologia, Pontifícia Universidade Católica de Campinas (Puc-Campinas)13087-571 Campinas - SP, Brasil https://orcid.org/0000-0003-4905-8476

\section{Resumo}

O envelhecimento populacional tem propiciado uma mudança na percepção dessa fase, a qual, mais recentemente, passou a ser visualizada como um período que pode ser vivenciado de forma saudável e com qualidade de vida. Nesse contexto a criatividade tem sido reconhecida como uma característica que pode contribuir para um envelhecimento bem-sucedido. Diante disso, o presente estudo buscou analisar a produção científica sobre criatividade e envelhecimento. Após busca nas bases de dados eletrônicas da Capes, Google Acadêmico, Pepsic, Redalyc, Research Gate e Scielo, 45 artigos foram selecionados. A análise dos trabalhos indicou um número pequeno de pesquisas, publicadas especialmente nos últimos anos. A maior parte dos artigos se mostrou de natureza empírica, envolvendo idosos entre participantes de outras idades. Diversas definições foram encontradas como base para os estudos sendo que, para a criatividade, a maior parte se baseia nas definições mais tradicionais (Torrance ou Guilford) enquanto para o envelhecimento, visões positivas e negativas dessa fase se fizeram presentes. Doze diferentes construtos foram investigados nas pesquisas.

Descritores: Criatividade; Idoso; Envelhecimento; Revisão.

\section{Abstract}

Population aging has led to a change in the perception of this phase, which, more recently, has come to be seen as a period that can be experienced in a healthy way and with quality of life. In this context, creativity has been recognized as a characteristic that can contribute to successful aging. Given this, the present study sought to analyze the scientific production of creativity and aging. After searching the Capes, Google Scholar, Pepsic, Redalyc, Research Gate and Scielo's electronic databases, 45 articles were selected. The analysis of the works indicated a small number of studies, published especially in recent years. Most of the articles proved to be empirical in nature, involving the elderly among participants of other ages. Several definitions were found as the basis for the studies. For creativity, most are based on the more traditional definitions (Torrance or Guilford), while for aging, positive and negative views of this phase were present. Twelve different constructs were investigated in the research.

Descriptors: Creativity; Aged; Aging; Review.

\section{Resumen}

El envejecimiento de la población ha supuesto un cambio en la percepción de esta fase que, más recientemente, pasó a ser vista como un período que se puede vivir de forma saludable y con calidad de vida. En este contexto, la creatividad ha sido reconocida como una característica que puede contribuir a un envejecimiento exitoso. Por tanto, este estudio buscó analizar la producción científica sobre creatividad y envejecimiento. Después de buscar en las bases de datos electrónicas de Capes, Academic Google, Pepsic, Redalyc, Research Gate y Scielo, se seleccionaron 45 artículos. El análisis de los trabajos indicó un número reducido de investigaciones, publicadas especialmente en los últimos años. La mayoría de los artículos eran de naturaleza empírica e incluían a personas mayores entre participantes de otras edades. Se encontraron varias definiciones como base para los estudios, y para la creatividad, la mayoría se basan en definiciones más tradicionales (Torrance o Guilford), mientras que para el envejecimiento, las opiniones positivas y negativas de esta fase estuvieron presentes. Se investigaron doce constructos diferentes en las encuestas.

Descriptores: Creatividad; Anciano; Envejecimiento; Revisión.

\section{INTRODUÇÃO}

A fase chamada de terceira idade ou velhice se inicia aos 60 anos e, no Brasil, essa parcela representa cerca de $13 \%$ da população'. Durante muito tempo o envelhecimento foi concebido como período de inevitável declínio, marcado por doença e senilidade. Mais recentemente, mudanças importantes vêm ocorrendo na compreensão dessa fase, de modo a reconhecer que o envelhecimento saudável exige muito mais do que condicionamento físico e prevenção de doenças $^{2}$. Tal conceito enfatiza a qualidade de vida, baseando-se em seis características: senso de propósito, interações com outras pessoas, crescimento pessoal, autoaceitação, autonomia e saúde ${ }^{3}$. Consequentemente, pesquisadores têm se atentado à importância de voltarem sua atenção não somente aos déficits que podem se fazer presentes nessa idade, mas também, aos aspectos sadios ${ }^{4}$.

O texto irá se focar na criatividade, característica que, dentre outras, vem sendo explorada, especialmente na última década, como um dos fatores que pode trazer vários benefícios para os idosos ${ }^{5}$ provocando impacto positivo na saúde física, mental e emocional $^{6}$. Consequentemente, a criatividade vem sendo valorizada como um elemento importante para 0 envelhecimento bemsucedido e manutenção da qualidade de vida nessa fase ${ }^{7}$.

Os benefícios da criatividade em idosos 
e seus impactos positivos no nível individual, comunitário e social ${ }^{8}$ vêm sendo notados, sugerindo mudanças de vida e novas perspectivas após o envolvimento em atividades dessa natureza ${ }^{9}$ especialmente em relação a ampliação das experiências de vida e bem-estar subjetivo, bem como promoção de sentimentos relacionados ao engajamento social, colaboração e sentimento de inclusão ${ }^{10}$.

Apesar do declínio das funções cognitivas, que é comum ao longo do envelhecimento, especialmente de algumas que são importantes para a expressão da criatividade, tais como velocidade de processamento, recuperação e memória a longo prazo, memória de trabalho e capacidade atencional $^{11}$, é nesta fase que as pessoas costumam ter mais tempo para se concentrarem em projetos de seu interesse e enfrentarem desafios pessoais.

Pesquisas têm demonstrado que alguns desses declínios não necessariamente irão gerar um efeito negativo. Segundo Carpenter, Chae e Yoon ${ }^{12}$, o aumento da distração, resultante da diminuição do controle inibitório, comum na velhice, tem mostrado efeitos positivos na criatividade na terceira idade. Pablo Picasso e Claude Monet $^{13}$ podem ser citados como exemplos de indivíduos que mantiveram sua produção criativa na velhice ${ }^{14-16}$.

Consequentemente, diversos estudos têm verificado efeitos positivos de intervenções baseadas na estimulação da criatividade em pessoas idosas, relacionados a aumento da autoconfiança, autoestima, sentimentos de domínio e realização, desenvolvimento de aspectos relacionados ao bem-estar, auxiliando o desenvolvimento de recursos de proteção para combater o risco de problemas de saúde mental ${ }^{17}$. Outros benefícios envolvem adaptação às mudanças na vida, aumento da saúde mental e possibilidade de se reinventar através da inserção de novas atividades criativas à sua rotina ${ }^{18}$, desenvolvimento de novas habilidades, motivação e resolução de problemas diários. A criatividade prática e diária, influencia positivamente a forma como os idosos gerenciam sua vida cotidiana, melhorando os índices de saúde física e mental, engajamento social, ampliando seu senso de competência, autoestima e a forma adaptativa como eles respondem às mudanças e situações adversas típicas dessa fase ${ }^{3}$. Para os idosos, a expressão criativa é uma forma de dividir suas estórias de vida, descobrir um potencial inexplorado ou continuar seu trabalho de vida, oferecendo alguma contribuição para suas comunidades ${ }^{19}$.

Consequentemente, os idosos podem demonstrar criatividade em níveis iguais aos apresentados por indivíduos mais jovens ${ }^{3}$ dadas algumas variáveis como mais tempo para praticar, entender e obter insights ${ }^{20}$. O autor considera que o despertar do potencial humano na segunda metade da vida, envolveria a criatividade aplicada a experiências de vida, na tentativa de inventar novas formas de viver, de modo que a maior parte das criações aconteceria mais tarde na vida.

De modo geral, os indivíduos criativos mais velhos teriam tendência a produções mais reflexivas, fruto de elaboração e procurariam sintetizar os valores tradicionais. Assim, as obras criadas tardiamente tendem a se apoiar na experiência subjetiva, sob um ponto de vista introspectivo, de modo a refletir suas experiências anteriores ${ }^{21}$, por meio do resgatar do passado e memórias pessoais como forma de expressão ${ }^{22}$. Na velhice, o pensamento criativo seria caracterizado como mais integrativo e relacionado à habilidade convergente, apresentando ótimo desempenho em relação a criatividade do dia a dia e no emprego de estratégias focadas na resolução de problemas ${ }^{23}$.

Apesar dos diversos benefícios que tal habilidade pode trazer para essa faixa etária, um número ainda reduzido de pesquisas sobre criatividade na terceira idade é encontrado ${ }^{24}$. Nesse sentido, a necessidade de realização de um maior número de estudos que tenham como foco tal construto entre pessoas na maturidade e terceira idade é recomendada ${ }^{25}$ sendo importante que um mapeamento das pesquisas existentes e, consequentemente, a identificação das lacunas seja feito para guiar investimentos direcionados a temáticas ainda pouco exploradas. Assim, o presente estudo teve como objetivo principal, analisar a produção científica nacional e internacional sobre criatividade e envelhecimento.

MATERIAL E MÉTODO

A fim de atingir o objetivo proposto neste estudo, foram realizadas buscas nas bases de dados eletrônicas Google acadêmico, Periódicos Eletrônicos de Psicologia (PePSIC), Rede de Revistas Científicas da América Latina e Caribe, Espanha e Portugal (Redalyc), Research Gate e Scientific Eletronic Library Online (SciELO). Buscou-se identificar artigos que tivessem, como tema, criatividade e envelhecimento. $O$ estudo seguiu as recomendações propostas pelo método PRISMA-P ${ }^{26}$.

- Estratégia de busca

As bases de dados eletrônicas foram consultadas em novembro de 2020, sem 
limitação de período. Os descritores "criatividade e envelhecimento", "criatividade e idoso", "criatividade e maturidade" e "criatividade e velhice" foram utilizados, separadamente, em português, inglês e espanhol. Os autores optaram por usar as terminologias mais comumente empregadas para descrever essa população.

- Critérios de Elegibilidade

Foram aplicados os seguintes critérios de elegibilidade: artigos que apresentavam a palavra "criatividade" no título ou assunto e que se referiam ao envelhecimento, idosos ou terceira idade. Foram excluídas teses e dissertações, trabalhos que não foram localizados na íntegra, citações, artigos que não enfocavam diretamente a temática da criatividade, artigos que não se referiam ao envelhecimento e aqueles que se encontravam duplicados. O Quadro 1 apresenta os resultados encontrados a partir da estratégia de busca adotada.

Quadro 1. Resultados das buscas realizadas nas bases de dados

\begin{tabular}{|c|c|c|c|c|c|c|c|c|c|c|c|c|c|c|c|}
\hline \multirow[t]{2}{*}{ Descritores } & \multicolumn{3}{|c|}{ Scielo } & \multicolumn{3}{|c|}{ Pepsic } & \multicolumn{3}{|c|}{ Redalyc } & \multicolumn{3}{|c|}{$\begin{array}{l}\text { Research } \\
\text { Gate }\end{array}$} & \multicolumn{3}{|c|}{$\begin{array}{c}\text { Google } \\
\text { Acadêmico }\end{array}$} \\
\hline & $T$ & $\mathrm{~S}$ & $\mathrm{R}$ & $\mathrm{T}$ & $\mathrm{S}$ & $\mathrm{R}$ & $T$ & $\mathrm{~S}$ & $\mathrm{R}$ & $\mathrm{T}$ & $\mathrm{S}$ & $\mathrm{R}$ & $\mathrm{T}$ & $\mathrm{S}$ & $\mathrm{R}$ \\
\hline $\begin{array}{l}\text { Criatividade e } \\
\text { envelhecimento }\end{array}$ & 7 & 2 & - & o & - & - & o & - & - & 1 & 1 & - & 11 & - & - \\
\hline $\begin{array}{ll}\text { Criatividade } & \mathrm{e} \\
\text { idoso } & \end{array}$ & 7 & - & - & 0 & - & - & 0 & - & - & 0 & - & - & 0 & - & - \\
\hline $\begin{array}{ll}\text { Criatividade } \\
\text { maturidade }\end{array}$ & 7 & 1 & 1 & 0 & - & - & o & - & - & 1 & - & 1 & 49 & - & - \\
\hline $\begin{array}{l}\text { Criatividade e } \\
\text { velhice }\end{array}$ & 3 & - & 2 & 1 & - & - & o & - & - & 1 & - & 1 & 1 & - & - \\
\hline $\begin{array}{l}\text { Creativity and } \\
\text { elderly }\end{array}$ & 18 & 2 & 2 & 0 & - & - & 1 & - & 1 & 3 & 3 & - & 5 & - & - \\
\hline $\begin{array}{l}\text { Creativity and } \\
\text { aging }\end{array}$ & 0 & - & - & 1 & - & - & 0 & - & - & 0 & - & - & 972 & 33 & - \\
\hline $\begin{array}{l}\text { Creativity and } \\
\text { old age }\end{array}$ & o & - & - & 1 & - & - & 4 & - & - & 7 & 2 & - & 42 & 1 & - \\
\hline $\begin{array}{l}\text { Creativity and } \\
\text { maturity }\end{array}$ & o & - & - & 1 & - & - & 0 & - & - & 3 & 1 & - & 218 & - & - \\
\hline $\begin{array}{l}\text { Creatividad y } \\
\text { envejecimiento }\end{array}$ & o & - & - & o & - & - & 0 & - & - & 0 & - & - & 10 & - & - \\
\hline $\begin{array}{l}\text { Creatividad y } \\
\text { anciano }\end{array}$ & 0 & - & - & 0 & - & - & 0 & - & - & 0 & - & - & 0 & - & - \\
\hline $\begin{array}{l}\text { Creatividad } \quad y \\
\text { madurez }\end{array}$ & 0 & - & - & 0 & - & - & 2 & - & - & 0 & - & - & 69 & - & - \\
\hline $\begin{array}{ll}\begin{array}{l}\text { Creatividad } \\
\text { vejez }\end{array} & \text { y } \\
\end{array}$ & 0 & - & - & 1 & - & - & 0 & - & - & 0 & - & - & 4 & - & - \\
\hline TOTAL & & 5 & & & o & & & o & & & 6 & & & 34 & \\
\hline
\end{tabular}

\section{- Registro de estudos}

Os registros foram feitos em planilha no programa Excel. A partir da leitura dos resumos dos artigos, por dois revisores independentes, uma triagem dos trabalhos foi realizada. Foram selecionados aqueles que atendiam ao foco da revisão sistemática. Posteriormente, os mesmos revisores atuaram nas demais fases, de elegibilidade, inclusão e análise.

\section{- Extração dos dados}

Após a aplicação dos critérios de inclusão e exclusão, 45 foram selecionados. Assim, para atingir o objetivo principal, as pesquisas foram analisadas quanto ao ano de publicação, participantes (idade e número), tipo de estudo (artigo empírico, teórico, estudo de caso, revisão de literatura), definição de envelhecimento ou idoso que embasa 0 trabalho, definição de criatividade que fundamenta a pesquisa, construtos investigados e instrumentos utilizados.

Os dados foram analisados estimandose a frequência e porcentagem de ocorrência de cada categoria de resposta. Nos casos em que um trabalho contemplava mais de uma categoria, a classificação foi feita em todas as categorias apresentadas no artigo, de modo que, em algumas delas, o número total de classificações ultrapassa o número de artigos analisados.

RESULTADOS E DISCUSSÃO

O percurso metodológico empregado na seleção dos artigos, segundo o método Prisma ${ }^{27}$ é apresentado na Figura 1. Após tal procedimento, 45 artigos foram selecionados, sendo cinco da Scielo, seis do Research Gate e 34 do Google Acadêmico.

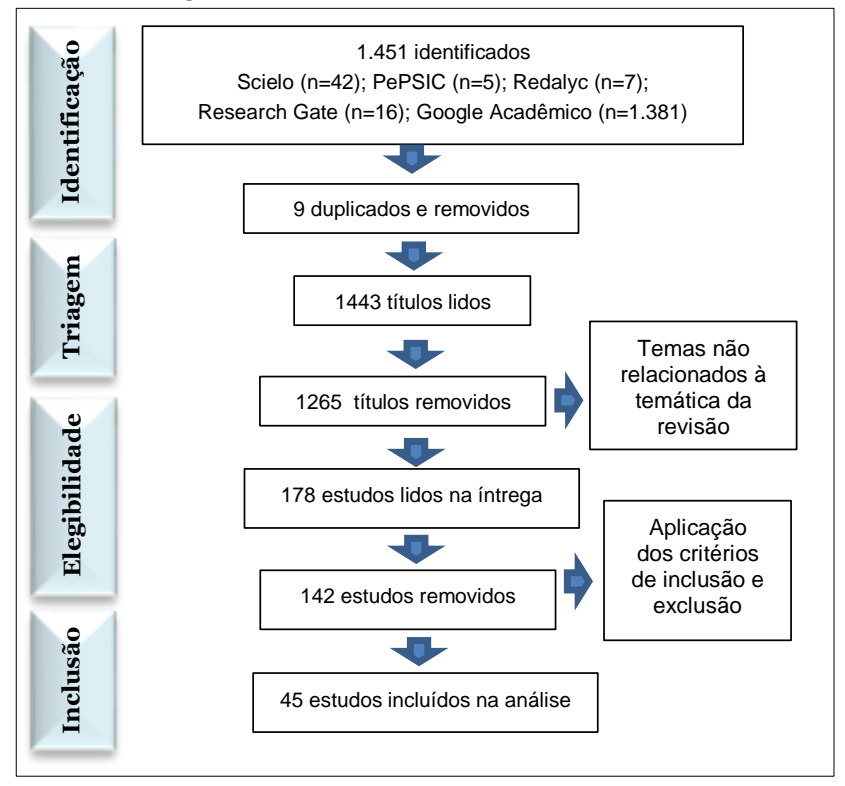

Figura 1: Fluxograma de busca e seleção dos artigos.

O primeiro dado analisado referiu-se ao ano de publicação dos artigos. O Gráfico 1 apresenta a distribuição de pesquisas que abordam criatividade na velhice, ao longo dos anos, visando-se compreender o interesse da literatura científica sobre a temática. Os resultados indicaram que o primeiro artigo encontrado foi publicado no ano de 1974 e, o mais recente, em 2020. A partir desses dados foi possível verificar o baixo interesse na investigação da criatividade na terceira idade, visto que no período estudado (37 anos), um total de 45 artigos foi localizado, indicando uma média de 1,21 artigo/ano publicado na temática. É importante ressaltar que, em diversos anos, nenhuma publicação foi encontrada.

O Gráfico indica que até 2006 o número de pesquisas sobre criatividade na terceira idade é bastante baixo, cerca de um artigo nos 
anos encontrados, sendo que vários anos não tiveram publicação na temática. Em 2006 um aumento no interesse pode ser notado, o qual, no entanto, não se mantém nos anos seguintes. Somente a partir de 2016 um crescimento mais constante pode ser visualizado. É importante ressaltar que uma queda aparente é visualizada no ano 2020, no entanto, é importante considerar que tal dado pode ter sido influenciado pelo fato das buscas terem sido realizadas antes do final do ano citado, de modo que diversos artigos publicados depois da consulta não foram incluídos na análise.

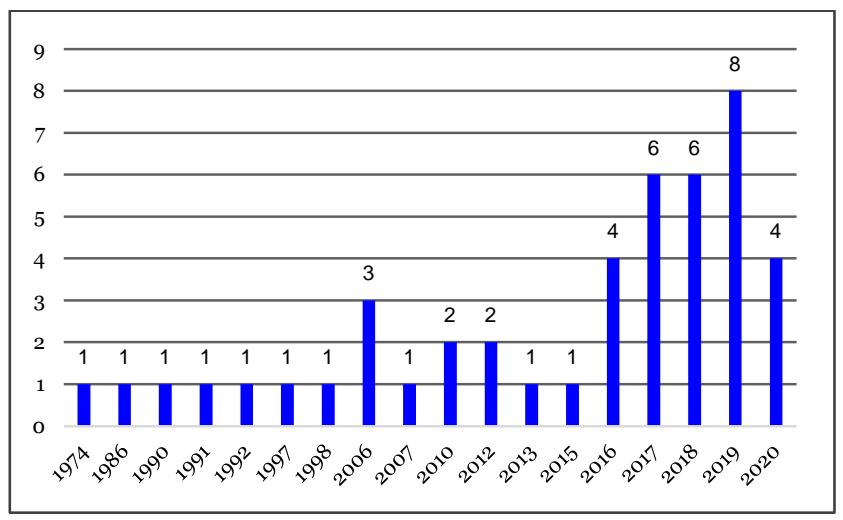

Gráfico 1: Distribuição de publicações por ano.

Em seguida foi investigado o tipo de estudo. Os dados indicaram que a maior parte dos estudos era de natureza empírica $(n=26$; $57,7 \%$ ), embora um número importante de estudos teóricos também tenha sido localizado $(n=16 ; 35,5 \%)$. Outros tipos encontrados foram pesquisa-ação, estudo de caso e revisão de literatura (um artigo cada).

Posteriormente, foi analisada a amostra dos artigos empíricos, considerando-se a idade e o número de participantes. Observou-se que dos 45 estudos, 20\% não forneceram informações sobre a idade dos participantes. Os demais apresentaram, na composição da amostra, idosos e participantes de outras idades, sendo que cinco pesquisas tiveram participantes com idade a partir de 20 anos, oito apresentaram amostra com idade a partir de 40 anos, dois contemplaram participantes com mais de 50 anos e 14 trabalhos avaliaram apenas a população com idade igual ou superior a 60 anos. Cabe ressaltar que nos estudos que a amostra foi composta apenas por idosos, a média de idade foi de 72 anos.

A partir dessa análise fica evidente que, embora haja um crescimento no número de estudos que abordem a criatividade na velhice, a maior parte das pesquisas sobre a temática não tem como foco principal a população idosa. É importante salientar que, embora os estudos investigassem a criatividade na velhice, grande parte das pesquisas optou em comparar esse construto em diferentes fases do desenvolvimento. Assim, é importante se fazer notar que, embora existam diferenças relacionadas à idade, todos os indivíduos possuem potencial criativo ${ }^{28}$. Neste sentido, Galenson $^{29}$, a partir da análise de realizações de expoentes na história defende que a criatividade não é um atributo exclusivamente jovem, pessoas mais velhas podem apresentar um desempenho excelente em atividades e produção criativa.

O número reduzido de estudos encontrado confirma os achados de outras revisões de literatura sobre criatividade. Em todos os casos, independente do contexto em que ela seja investigada, é possível verificar que a população idosa praticamente não tem sido contemplada nos estudos ${ }^{30-33}$. Neste sentido, a necessidade de realização de um maior número de estudos que tenham como foco a criatividade entre pessoas na maturidade e terceira idade é recomendada ${ }^{25}$.

De modo geral, a investigação da criatividade e envelhecimento têm apresentado resultados divergentes. Enquanto algumas pesquisas enfatizam que a criatividade diminui com a idade, devido principalmente aos declínios na memória de trabalho, outras afirmam que dependendo da definição e medida de criatividade, os idosos podem demonstrar criatividade igual aos níveis apresentados por indivíduos mais jovens ${ }^{3}$. Além disso, o tipo de criatividade também se mostraria diferente nessa idade, sendo marcada por produções mais reflexivas e introspectivas, apoiadas na experiência subjetiva, nas experiências anteriores do sujeito ${ }^{21}$, baseada na resolução de problemas do dia a dia e no emprego de estratégias focadas na resolução de problemas $^{23}$. É comum encontrar exemplos de pessoas que alcançaram seu auge criativo na velhice ${ }^{34}$.

Considerando-se que os dois construtos investigados não apresentam consenso em relação à sua definição e que a multiplicidade de definições para um mesmo construto pode influenciar diretamente os resultados encontrados nas pesquisas, em seguida buscou-se compreender as definições que embasavam os estudos analisados. Para tanto, analisou-se as definições utilizadas pelos autores para abordar tanto a criatividade, quanto o envelhecimento, apresentadas em ordem cronológica de publicação (Quadro 2) 4,5,9,22,25,35-56. Cabe ressaltar que quanto a definição de criatividade, foram analisados apenas os artigos empíricos, relatos de experiência e pesquisa-ação. 
Quadro 2. Definição dos Construtos Envelhecimento e Criatividade.

\begin{tabular}{|c|c|c|}
\hline Autor & $\begin{array}{c}\text { Definição de } \\
\text { envelhecimento }\end{array}$ & Definição de criatividade \\
\hline $\begin{array}{l}\text { Adams-Price } \\
\text { et al. (2018) }\end{array}$ & $\begin{array}{l}\text { Envelhecimento bem } \\
\text { sucedido, definido como } \\
\text { um indicador de saúde e } \\
\text { boa adaptação ao processo } \\
\text { de envelhecimento }\end{array}$ & $\begin{array}{l}\text { Hobby criativo: participação repetida em uma } \\
\text { atividade expressiva específica que é } \\
\text { pessoalmente significativa e oferece } \\
\text { oportunidades para crescimento, aprendizagem } \\
\text { e desenvolvimento de habilidades }\end{array}$ \\
\hline $\begin{array}{l}\text { Adnan et al. } \\
\text { (2019) }\end{array}$ & Não apresenta & Habilidade de produzir algo novo e útil \\
\hline $\begin{array}{l}\text { Alforque } \\
(2017)\end{array}$ & Não apresenta & $\begin{array}{l}\text { Característica humana que empodera pessoas e } \\
\text { comunidades a se adaptarem e florescerem } \\
\text { diante de mudanças de circunstâncias }\end{array}$ \\
\hline $\begin{array}{l}\text { Cantu } \\
\text { Fleurie } \\
(2018)\end{array}$ & Não Apresenta & $\begin{array}{l}\text { Concepção holística: a criatividade desempenha } \\
\text { função curativa e é expressa a partir de } \\
\text { atividades de arte, como pintura e escrita. }\end{array}$ \\
\hline $\begin{array}{l}\text { Cera et al. } \\
(2018)\end{array}$ & Não Apresenta & Não Apresenta \\
\hline $\begin{array}{l}\text { Colombo et } \\
\text { al. (2018) }\end{array}$ & Não apresenta & $\begin{array}{llll}\text { Habilidade } & \text { composta } & \text { por três operações } \\
\text { mentais: } & \text { abertura, } & \text { conectividade } \\
\text { reorganizacão }\end{array}$ \\
\hline $\begin{array}{l}\text { Dohr } \\
\text { Forbess } \\
\text { (1986) }\end{array}$ & Não apresenta & $\begin{array}{l}\text { Abordagem Cognitiva de Guilford (1967): } \\
\text { características cognitivas como fluência, } \\
\text { flexibilidade, elaboração e originalidade são } \\
\text { consideradas básicas ou essenciais para a } \\
\text { criatividade. }\end{array}$ \\
\hline $\begin{array}{l}\text { Escalona et } \\
\text { al. (2017) }\end{array}$ & $\begin{array}{|lrr|}\text { Processo } & \text { marcado } & \text { por } \\
\text { declínios } & \text { físicos } & \text { e } \\
\text { cognitivos, } & \text { perdas } & \text { em } \\
\text { diversos } & \text { aspectos } & \text { que } \\
\text { podem } & \text { culminar } & \text { em } \\
\text { psicopatologias. } & \\
\end{array}$ & $\begin{array}{l}\text { Processo subjetivo, tanto individual como } \\
\text { social, onde se o indivíduo se mostra como } \\
\text { sujeito. }\end{array}$ \\
\hline Flood (2006) & \begin{tabular}{|lrr} 
Estar saudável e ativo, \\
considerando as dimensões \\
física, cognitiva e social, \\
apesar das perdas e \\
declínios próprios do do \\
avanço da & didade \\
(Envelhecimento & Bem \\
Sucedido/Positivo) & \\
\end{tabular} & $\begin{array}{l}\text { Torrance (1965): habilidade de identificar } \\
\text { problemas, deficiências, lacunas buscar por } \\
\text { soluções, formular hipóteses testar hipóteses, } \\
\text { comunicar os resultados. }\end{array}$ \\
\hline $\begin{array}{l}\text { Gutheil e } \\
\text { Heyman } \\
(2016)\end{array}$ & Não apresenta & $\begin{array}{l}\text { Criatividade Artística como forma de promover } \\
\text { o bem estar e habilidades cognitivas }\end{array}$ \\
\hline $\begin{array}{l}\text { Lindauer et } \\
\text { al. (1997) }\end{array}$ & Não apresenta & $\begin{array}{l}\text { Criatividade artística: capacidade do indivíduo } \\
\text { de criar obras com valor e com elevado grau de } \\
\text { diferenciação em relação a outras. }\end{array}$ \\
\hline $\begin{array}{l}\text { Lorenzen- } \\
\text { Huber (1991) }\end{array}$ & Não apresenta & $\begin{array}{l}\text { Torrance (1965): habilidade de identificar } \\
\text { problemas, deficieincias, lacunas buscar por } \\
\text { soluções, formular hipóteses testar hipóteses, } \\
\text { comunicar os resultados. }\end{array}$ \\
\hline $\begin{array}{l}\text { Maduro } \\
\text { (1974) }\end{array}$ & $\begin{array}{l}\text { Período de transição e } \\
\text { transcendência de questões } \\
\text { egóicas, que implica em } \\
\text { ceder lugar ao mais jovem, } \\
\text { de forma que seus } \\
\text { conhecimentos sano } \\
\text { passados paras as geraçôes. }\end{array}$ & $\begin{array}{l}\text { Teoria folclórica hindu: A criatividade é um } \\
\text { dom divino, ou forma de conexão com deuses. } \\
\text { Reconhece o pensamento divergente como } \\
\text { forças de deuses. }\end{array}$ \\
\hline es et al. & ão apresenta & \\
\hline $\begin{array}{l}\text { Mendonça e } \\
\text { Coutinho } \\
\text { (2019) }\end{array}$ & Não apresenta & $\begin{array}{l}\text { Teoria humanista de Criatividade artística de } \\
\text { Rollo May }\end{array}$ \\
\hline $\begin{array}{l}\text { Nakano et al. } \\
(2019)\end{array}$ & $\begin{array}{l}\text { Processo r dinâmico, } \\
\text { progressivo, caracterizado } \\
\text { por diversas modificações } \\
\text { biológicas, psíquicas e e } \\
\text { sociais. }\end{array}$ & $\begin{array}{l}\text { Torrance (1965): habilidade de identificar } \\
\text { problemas, deficieincias, lacunas buscar por } \\
\text { soluçôes, formular hipóteses testar hipóteses, } \\
\text { comunicar os resultados. }\end{array}$ \\
\hline $\begin{array}{l}\text { Nuessel et al. } \\
\text { (2010) }\end{array}$ & Não Apresenta & $\begin{array}{l}\text { Criatividade artística embasada na teoria } \\
\text { humanista proposta por Rollo May: } \\
\text { compreendida como manifestação básica de um } \\
\text { homem realizando o seu eu no mundo. }\end{array}$ \\
\hline $\begin{array}{l}\text { Palmiero et } \\
\text { al. (2017). }\end{array}$ & Não apresenta & Não apresenta \\
\hline $\begin{array}{l}\text { Pandya } \\
(2020)\end{array}$ & Não apresenta & $\begin{array}{l}\text { Abordagem Cognitiva de Guilford (1967): } \\
\text { características cognitivas como fluência, } \\
\text { flexibilidade, elaboração e originalidade são } \\
\text { consideradas básicas ou essenciais para a } \\
\text { criatividade. }\end{array}$ \\
\hline $\begin{array}{l}\text { Reynolds et } \\
\text { al. (2016) }\end{array}$ & $\begin{array}{l}\text { Apesar de ser uma fase } \\
\text { cercada de problemas, pode } \\
\text { ser visualizada um período } \\
\text { de desenvolvimento }\end{array}$ & $\begin{array}{l}\text { Criatividade Artística como ferramenta para o } \\
\text { crescimento pessoal }\end{array}$ \\
\hline Sabeti (2015) & $\begin{array}{l}\text { Envelhecimento } \\
\text { Sucedido/Positivo }\end{array}$ & $\begin{array}{l}\text { ida como a capacidade de } \\
\text { que já foi vivido e a partir } \\
\text { futuro }\end{array}$ \\
\hline $\begin{array}{l}\text { Sharma e } \\
\text { Babu (2017) }\end{array}$ & Não apresenta & $\begin{array}{l}\text { Fenômeno complexo e multifacetado que } \\
\text { envolve o desenvolvimento de uma nova ideia, } \\
\text { produto ou solução para um problema, e que } \\
\text { apresenta relevância para o sujeito, grupo ou } \\
\text { sociedade }\end{array}$ \\
\hline $\begin{array}{l}\text { Souza } \quad \text { e } \\
\text { Wechsler } \\
\text { (2013) }\end{array}$ & Não apresenta & $\begin{array}{l}\text { Torrance (1965): habilidade de identificar } \\
\text { problemas, deficiências, lacunas buscar por } \\
\text { soluçôes, formular hipóteses testar hipóteses, } \\
\text { comunicar os resultados. }\end{array}$ \\
\hline $\begin{array}{lr}\text { Smith e Van } \\
\text { Der } & \text { Meer } \\
(1990) & \end{array}$ & Não apresenta & $\begin{array}{l}\text { Teoria de Pruyser (1987): uma atitude em } \\
\text { relação à vida, que pode ou não estar associada } \\
\text { ao talento artístico, originalidade científica, ou } \\
\text { outros traços criativos. }\end{array}$ \\
\hline $\begin{array}{l}\text { Trnka et al. } \\
\text { (2019) }\end{array}$ & Não apresenta & $\begin{array}{l}\text { Torrance (1965): habilidade de identificar } \\
\text { problemas, deficiências, lacunas buscar por } \\
\text { soluções, formular hipóteses testar hipóteses, } \\
\text { comunicar os resultados. }\end{array}$ \\
\hline Wiggs (2010) & $\begin{array}{l}\text { senvolvimento interno e } \\
\text { scimento contínuo do eu } \\
\text { eoria do envelhecimento } \\
\text { Jung) }\end{array}$ & $\begin{array}{l}\text { Criatividade artística Junguiana: a criatividade } \\
\text { é concebida como um complexo autônomo. }\end{array}$ \\
\hline $\begin{array}{l}\text { Zavarize e } \\
\text { Wechsler } \\
\text { (2012) }\end{array}$ & Não apresenta & $\begin{array}{l}\text { Torrance (1965): habilidade de identificar } \\
\text { problemas, deficiências, lacunas buscar por } \\
\text { soluções, formular hipóteses testar hipóteses, } \\
\text { comunicar os resultados. }\end{array}$ \\
\hline
\end{tabular}

De modo geral, foi possível verificar a predominância de definições tradicionais para a criatividade, tais como aquelas elaboradas por Torrance ou Guilford. No entanto, diferentes definições também foram encontradas, compreendendo o construto dentro de teorias menos frequentemente utilizadas na criatividade, tais como folclórica hindu, Teoria de Pruyser, Teoria Humanista, Junguiana dentro de uma concepção holística. Ainda foram encontrados estudos enfocando tipos específicos de criatividade, tal como a artística. Por outro lado, o envelhecimento foi visualizado tanto como um período de transição, processo dinâmico marcado por modificações em diversos aspectos, como uma fase marcada por ganhos e uma compreensão positiva, quanto marcada por declínios, dentro de um caráter negativo. É importante ressaltar que a maior parte dos estudos não apresentou as definições que os embasam.

Por fim, considerando-se que é comum que os estudos sobre criatividade na velhice envolvam outros construtos ${ }^{4,25,48}$, 0 levantamento dessa informação foi efetuado, assim como os instrumentos utilizados para avaliar cada um deles. Os resultados são apresentados no Quadro 3. Nessa análise os artigos teóricos foram excluídos, sendo importante destacar que alguns estudos fizeram uso de mais de um instrumento, desta forma, foi contabilizada cada ocorrência.

Quadro 3. Construtos Investigados e Instrumentos Utilizados.

\begin{tabular}{|c|c|}
\hline Construtos & Instrumentos \\
\hline \multirow[t]{8}{*}{ Criatividade } & Pensando Criativamente com Palavras de Torrance \\
\hline & Pensando Criativamente com Figuras de Torrance \\
\hline & Creative Benefits Scale \\
\hline & Emotional Creativity Inventory \\
\hline & Escala de Estilos de Pensar e Criar \\
\hline & Escala do Perfil Criativo \\
\hline & Runco Ideational Behavior Scale (RIBS) \\
\hline & The Creativity Test (ProCriativ) \\
\hline Qualidade de Vida & Whoqol-bref \\
\hline \multirow{6}{*}{ Inteligência/ Cognição } & Bateria de Habilidades Intelectuais de Adultos \\
\hline & Frontal Systems Behavior Scale \\
\hline & Stroop Test NIMHANS \\
\hline & Subtest PGI memory scale \\
\hline & Wechsler Adult Intelligence Scale (WAIS) \\
\hline & Mini Mental State Examination \\
\hline Estresse & Escala de Estresse Percebido \\
\hline \multirow{2}{*}{$\begin{array}{l}\text { Auto percepção de estado de } \\
\text { Saúde }\end{array}$} & Escala Visual Analógica \\
\hline & Identification Test (IT) \\
\hline \multirow[t]{2}{*}{ Estado de Saúde Mental } & Escala de Saúde Mental Positiva \\
\hline & Mental Balance \\
\hline Motivação & Motivational Participation Inventor \\
\hline \multirow[t]{2}{*}{ Personalidade } & Personality Trait Inventory (WI) \\
\hline & The Meta-Contrast Technique (MCT) \\
\hline \multirow[t]{6}{*}{ Bem- estar/Satisfação com a Vida } & Escala de Satisfação com a vida \\
\hline & Satisfaction with Life Scale \\
\hline & Life Satisfaction Inventory \\
\hline & Purpose in Life Test \\
\hline & Rotter's IE Locus of Control instrument \\
\hline & The Philadelphia Geriatric Center Morale Scale \\
\hline \multirow[t]{2}{*}{ Depressão } & Geriatric Depression Scale Short Form \\
\hline & Loneliness Scale UCLA \\
\hline Generatividade & Loyola Generativity Scale (LGS) \\
\hline $\begin{array}{l}\text { Disposição para pensamento } \\
\text { Lateral }\end{array}$ & Lateral Thinking Disposition Scale (LATD) \\
\hline \multirow[t]{2}{*}{ Memória } & Cognitive Complaint Interview \\
\hline & PGI Memory Scale \\
\hline Concepção de aprendizado & Popular Conceptions of Learning \\
\hline \multirow{2}{*}{ Reserva Cognitiva } & Cognitive Reserve Questionnaire \\
\hline & Cognitive Reserve Test \\
\hline Função Executiva & Stroop Test \\
\hline
\end{tabular}


A partir do Quadro 3 é possível verificar que dezesseis diferentes construtos foram investigados nas pesquisas. Em relação à criatividade, construto foco da revisão aqui apresentada, oito diferentes instrumentos foram utilizados, sendo dois nacionais (Escala de Estilos de Pensar e Criar e Escala do Perfil Criativo) e outros seis internacionais. Destes, somente a Escala de Estilos de Pensar e Criar se encontra disponível para uso profissional.

Os demais construtos avaliados na terceira idade envolveram aspectos mais gerais, como inteligência/cognição e personalidade, e aspectos positivos mais específicos (qualidade de vida, motivação, bem-estar/satisfação com a vida). Possivelmente estes últimos se baseiam na compreensão do envelhecimento como um processo que pode ser visualizado dentro de uma perspectiva positiva. No entanto também foram encontrados estudos voltados à investigação de aspectos negativos que podem se fazer presentes nessa população, tais como estresse ou depressão. Outros construtos como estado de saúde mental e percepção do estado de saúde podem ter sido investigados nas duas visões, na busca por pontos fortes ou fracos nos idosos.

\section{CONSIDERAÇÕES FINAIS}

Diante dos resultados obtidos neste estudo foi possível observar que, embora o reconhecimento da importância da criatividade na terceira idade venha se mostrando cada vez mais presente na literatura científica, a quantidade de pesquisas sobre essa temática ainda é reduzida, especialmente no contexto brasileiro.

A mudança de compreensão do processo de envelhecimento, como uma fase que pode ser vivenciada de maneira saudável e positiva tem englobado, dentre os vários aspectos, a criatividade como um diferencial. A revisão dos estudos evidenciou que a criatividade pode não apenas ser estimulada e desenvolvida na terceira idade, como também pode ser um fator que contribuiu para o alcance do envelhecimento bem sucedido. A substituição daquela compreensão de velhice como uma fase marcada somente por perdas se mostra urgente, a fim de que políticas voltadas à manutenção do bem-estar dos idosos possam ser planejadas e oferecidas para essa parcela da população cada vez maior.

Como limitações da pesquisa, alguns fatores, tais como a seleção das bases de dados, bem como a limitação de acesso a versão eletrônica das bases pode ter atuado de modo a impedir que alguns trabalhos não tenham sido localizados na busca realizada. Do mesmo modo a seleção dos operadores boleanos e a impossibilidade de acesso a alguns textos completos também devem ser citados como possíveis vieses da revisão. No entanto, apesar dos possíveis vieses citados, a avaliação dos estudos selecionados apontou para tendências presentes na temática, bem como as lacunas ainda encontradas, especialmente aquelas relacionadas à ausência das definições dos conceitos (criatividade $e$ envelhecimento) e de medidas adequadas para uso na população brasileira e nessa faixa etária específica.

REFERÊNCIAS

1. Instituto Brasileiro de Geografia e Estatística (2020). Censo 2020: idosos indicam caminhos para uma melhor idade. Disponível em: https://censo2020.ibge.gov.br/2012-agencia-denoticias/noticias/24036-idosos-indicamcaminhos-para-uma-melhor-idade.html.

2. Larson RTH, Perlstein S. Creative aging: a new filed for 21st century. Teaching Artist Journal. 2003;1(3):144-51.

3. McHugh MC. Experiencing flow: creativity and meaningful task engagement for senior women. Women Ther. 2016;39(3-4):280-95.

4. Nakano TC, Machado WL, Abreu ICC. Relações entre estilos de pensar e criar, bemestar, saúde percebida e estresse na terceira idade. Psico-USF. 2019;24(3), 555-68.

5. Palmiero M, Nori R, Piccardi L. Verbal and visual divergent thinking in aging. Exp Brain Res. 2017;235:1021-29.

6. Behroozi M, Manesh MA, Fadaiyan B, Behroozi $S$. Investigation of relationship among creativity, spiritual intelligence, perfectionism and mental health of bushehr artists. Procedia: Soc Behav Sci. 2014;143:399-403.

7. Cristini $C$, Cesa-Bianchi M. Culture, creativity and quality of life in old age. In: Bianco A, Conigliaro P, Gnaldi M (Eds.), Italian Studies on Quality of Life. Social Indicators Research Series (pp. 243-253). Springer, Cham; 2019.

8. Mental Health Foundation. An evidence review of the impact of participatory arts on older people. 2011. Disponível em: http://www.mentalhealth.org.uk/publications/evi dence-review-participatory-arts-older-people/

9. Gutheil IA, Heyman JC. Older adults and creative arts: personal and interpersonal change. Act Adapt Aging. 2016; 40(3):169-79.

10. Creech A, Larouche K, Generale M, Fortier D. Creativity, music, and quality of life of later life: a systematic review. Psychology of Music. 2020:1-21.

11. Fischler IS, Heilman KM. Brain aging and creativity. In: Heilman KM, Nadeau SE (Eds.), Cognitive changes and the aging brain (pp. 
188-202). Cambridge: Cambridge University Pres; 2019.

12. Carpenter SM, Chae RL, Yoon C. Creativity and aging: Positive consequences of distraction. Psychol Aging. 2020;35(5):654-62.

13. Llorente M, Parra FC, McGuire M. (2017). Productive aging and creativity: the lives and art of George O'keefe, Pablo Picasso and Claude Monet. Am J Geriatr Psychiatry. 2017;25(3).

14.Domínguez-Rué $E$. The art of doing good. Aging, creativity and wisdom in the Isabel Dalhousie novels. J Aging Stud. 2018;44:22-7.

15. Gogol M. Late painting - creativity during aging and disease. Innov Aging. 2018; 2(1):459.

16. Kozak A, Parra FC, Llorente M. Productive aging and creativity: the lives and art of M. C. Escher, Agnes Martin, Yayo Kusama and Carmen Herrera. Am J Geriatr Psychiatry. 2019;27(3):S47.

17. Ray M. Creativity and the Arts for Older People Living with Depression. In: Chew-Graham C, Ray M (Eds.) Mental Health and Older People. Springer, Cham; 2016.

18. Duhamel KV. Creativity and the golden years: biopsychosocial and cultural influences for living a successful life. Sociol Anthropol. 2016;4:1093-98.

19. Kaufman J, Finkelstein R. Creative aging in NYC. New York: Brookdale Center for Health Aging, Life Time Arts and Live on NY. Disponível em: https://brookdale.org/wpcontent/uploads/2020/02/Creative-Aging-inNew-York_FINAL.pdf.

20. Hanna GP. The central role of creative aging. J Art Life. 2013;4(1):1-15.

21. Lubart T. Psicologia da criatividade. Porto Alegre: Artmed; 2007.

22. Sabeti S. Creative ageing? Selfhood, temporality and the older adult learner. International J Life Long Educ. 2015;34(2): 211-29.

23. Hui ANN, He MWJ, Wong W. (2019). Understanding the development of creativity across the life span. In: Kaufman JC, Sternberg RJ (Eds.). The Cambridge Handbook of Creativity. United Kingdom: Cambridge University Press; 2019

24. Souza AAF de. Inteligência e Criatividade na Maturidade e Velhice [tese]. Campinas: Pontifícia Universidade Católica de Campinas; 2011

25. Souza AAF, Wechsler SM. Inteligência e criatividade na maturidade e velhice. Psicol: Reflex Crít. 2013;26(4):643-53.

26. Moher D, Shamseer L, Clarke M, Ghersi D, Liberati $A$, Petticrew $M$ et al. Preferred reporting items for systematic review and meta-analysis protocols (PRISMA-P) 2015 statement. Syst Rev. 2015;4(1):1-9.
27. Moher D, Liberati A, Tetzlaff J, Altman DG. Preferred reporting items for systematic reviews and meta-analyses: The PRISMA Statement. PLoS Med. 2009;6(7):e1000097.

28. Wechsler SM. Criatividade: descobrindo e encorajando. 3 ed. Campinas: Impressão Digital do Brasil; 2008.

29. Galenson DW. The nature of creativity in old age. Becker Friedman Institute for Economics Working Paper, 2019-67; 2019.

30. Alves RJR, Nakano TC. Dupla excepcionalidade: relações entre altas habilidades/superdotação com a síndrome de Asperger, Transtorno de Déficit de Atenção e Hiperatividade e Transtornos de Aprendizagem. Rev psicopedag. 2015;32(99):346-60.

31. Nakano TC, Wechsler SM. Criatividade: características da produção científica brasileira. Aval psicol. 2007;6(2):261-70.

32. Silva TF, Nakano TC. Criatividade no contexto educacional: análise de publicações periódicas e trabalhos de pós-graduação na área da Psicologia. Educ Pesq. 2012;38(3):743-59.

33. Wechsler SM, Nakano TC. Caminhos para a avaliação da criatividade: perspectiva brasileira. In: Primi R (Org.). Temas em Avaliação Psicológica. Campinas: Impressão Digital do Brasil; 2002.

34. Amendoeira MCR. A criatividade como expressão da subjetividade no envelhecimento. Pan Am J Aging Res. 2017;5(2):35-40.

35. Adams-Price CE, Nadorff DK, Morse LW, Davis KT, Stearns MA. The Creative Benefits Scale: connecting generativity to life satisfaction. Int $\mathrm{J}$ Aging Hum Dev. 2018;86(3):242-65.

36. Adnan A, Beaty R, Lam J, Spreng RN, Turner GR. Intrinsic default: executive coupling of the creative aging brain. Soc Cog Affec Neurosci. 2019;14(3):291-303.

37. Alforque MLG. Being less is never least unraveling creativity among older adults with disability. Malays J Med Res. 2017;1(2):1-7.

38. Cantu AG, Fleuriet KJ. Making the Ordinary More Extraordinary": Exploring Creativity as a Health Promotion Practice Among Older Adults in a Community-Based Professionally Taught Arts Program. J Holist Nurs. 2018;36(2):123-33.

39. Cera R, Cristini C, Antonietti A. Conceptions of learning, well-being, and creativity in older adults. Educ Cult Psychol Stud.2018;18:241-74.

40. Colombo B, Antonietti A, Daneau B. The relationships between cognitive reserve and creativity. a study on american aging population. Front Psychol. 2018;9:764.

41. Dohr JH, Forbess LA. Creativity, arts, and Profiles of aging: a reexamination. Educ Gerontol. 1986;12(2):123-38.

42. Escalona MS, Laffita DM, Pérez CY, Leyva D. C. Evaluación de indicadores funcionales 
asociados a la creatividad en el adulto mayo. MEDISAN. 2017;21(11):3136-44.

43. Flood M. Exploring the relationships between creativity, depression, and successful aging. Act Adapt Aging. 2007;31(1):55-71.

44. Lindauer MS, Orwoll L, Kelley MC. Aging artists on the creativity of their old age. Creat Res J. 1997;10(23):133-52.

45. Lorenzen-Huber L. Self-perceived creativity in the later Years: case studies of older nebraskans. Educ Gerontol: Int Quart. 1991;17(4):379-90.

46. Maduro R. Artistic Creativity and Aging in India. Int J Aging Hum Dev. 1974;5(4):303-29.

47. Mendes A, Câmara J, Caires AL, Garcês S, Pocinho M. Creativity and cognitive reserve in old age: an exploratory study in the Portuguese population. Rev Psicol. 2020;34(1):229-35.

48. Mendonça BIO, Coutinho DMB. Processo criativo e envelhecimento em uma pesquisaação. Rev Psicol. 2019;28(1):1-13.

49. Nuessel F, Van Stewart A, Cedeño A. A course on humanistic creativity in later life: literature review, case histories, and recommendations. Educ Gerontol. 2001;27(8):697-715.

50. Pandya SP. Meditation to improve lateral and divergent thinking among older adults: a 2-year follow-up study. Aging Clin Exp Res. 2020; 32:723-32.

51. Reynolds J, Bernard, Rezzano, M. J., \& Rickett M. Ageing, drama, and creativity: translating research into practice. Gerontol Geriatr Educ. 2016:37(3):307-28.

52. Sharma S, Babu N. Interplay between Creativity, Executive Function and Working Memory in Middle-Aged and Older Adults. Creat Res J. 2017;29(1):71-7.

53. Smith GJW, Van der Meer G. Creativity in old age. Creat Res J. 1990;3(4):249-64.

54. Trnka R, Cabelkova I, Kuska M, Nikolai T. Cognitive decline influences emotional creativity in the elderly. Creat Res J. 2019;31(1):93-101.

55. Wiggs CM. Creating the Self: Exploring the life journey of late-midlife women. J Women Aging. 2010;22(3):218-33.

56.Zavarize SF, Wechsler SM. Perfil criativo e qualidade de vida: implicações em adultos e idosos com dor lombar crônica. Rev Bras Geriatr Gerontol. 2012;15(3):403-14.

\section{CONFLITO DE INTERESSES}

Os autores declaram não haver conflitos de interesse

AUTOR PARA CORRESPONDÊNCIA

\section{Tatiana de Cássia Nakano}

Programa de Pós-Graduação em Psicologia, Pontifícia Universidade Católica de Campinas (Puc-Campinas)

13087-571 Campinas - SP, Brasil

E-mail: tatiananakano@hotmail.com 